\title{
On the CR transversality of holomorphic maps into hyperquadrics
}

Dedicated to Professor Yum-Tong Siu on the occasion of his 70th birthday

Xiaojun Huang and Yuan Zhang

\begin{abstract}
Let $M_{\ell}$ be a smooth Levi-nondegenerate hypersurface of signature $\ell(0<$ $\ell<\frac{n-1}{2}$ ) in $\mathbf{C}^{n}$ with $n \geq 3$, and write $H_{\ell}^{N}$ for the standard hyperquadric of the same signature in $\mathbf{C}^{N}$ with $N-n<\frac{n-1}{2}$. Let $F$ be a holomorphic map sending $M_{\ell}$ into $H_{\ell}^{N}$. Assume $F$ does not send a neighborhood of $M_{\ell}$ in $\mathbf{C}^{n}$ into $H_{\ell}^{N}$. We show that $F$ is necessarily CR transversal to $M_{\ell}$ at any point. Equivalently, we show that $F$ is a local CR embedding from $M_{\ell}$ into $H_{\ell}^{N}$.
\end{abstract}

\section{Introduction and the main theorems}

Let $M_{1}$ and $M_{2}$ be two connected smooth CR hypersurfaces in $\mathbf{C}^{n}$ and $\mathbf{C}^{N}$, respectively, with $3 \leq n \leq N$. Let $F$ be a holomorphic map from some small neighborhood $U \subset \mathbf{C}^{n}$ of $M_{1}$ into $\mathbf{C}^{N}$ with $F\left(M_{1}\right) \subset M_{2}$. Given a point $p \in M_{1}$, denote by $T_{p}^{(1,0)} M_{1}$ and $T_{F(p)}^{(1,0)} M_{2}$ the holomorphic tangent vector spaces of $M_{1}$ at $p$ and $M_{2}$ at $F(p)$, respectively. Assume $F$ does not send a neighborhood of $p$ in $\mathbf{C}^{n}$ into $M_{2}$. An important question in the study of the geometric structure of $F$ is to understand the geometric conditions for the manifolds in which $F$ is CR transversal to $M_{1}$ at $p$. Recall that $F$ is said to be CR transversal at $p$ if

$$
T_{F(p)}^{(1,0)} M_{2}+d F\left(T_{p}^{(1,0)} \mathbf{C}^{n}\right)=T_{F(p)}^{(1,0)} \mathbf{C}^{N}
$$

Xiaojun Huang, partially supported in part by National Science Foundation DMS-1363418.

Department of Mathematics, Rutgers University, New Brunswick, NJ 08903, USA e-mail: huangx@math.rutgers.edu

Yuan Zhang, partially supported in part by National Science Foundation DMS-1265330.

Department of Mathematical Sciences, Indiana University - Purdue University, Fort Wayne, IN 46805, USA e-mail: zhangyueipfw.edu 
Roughly speaking, the CR transversality property can be interpreted as an nonvanishing property of the normal derivative of the normal components for the map.

The problem has been extensively investigated in the literature. When both the target and the source manifolds are strongly pseudoconvex, CR transversality always holds due to the classical Hopf lemma. In the equal dimensional case $(n=N)$, work has been done by Pinchuk [Pi], Fornaess [Fo], Baouendi-Rothschild [BR], Ebenfelt-Rothschild [ER], Huang [Hu2], Isaev [Iz1] [Iz2], Huang-Pan [HP] and the references therein. The study of the higher codimensional case starts with the work of Baouendi-Huang in $[\overline{\mathrm{BH}}]$ where it is proved that the CR transversality always holds when the manifolds are hyperquadrics of the same signature. Baouendi-Ebenfelt-Rothschild [BER2] proved, under rather general setting, that the CR transversality holds in an open dense subset. See also a recent paper of EbenfeltSon $[\mathrm{ES}]$ and the references therein.

While there exist examples where CR transversality fails on certain thin sets (see, for instance [BER2]), as mentioned above, the rigidity theorem due to BaouendiHuang $[\overline{\mathrm{BH}}]$ indicates that the $\mathrm{CR}$ transversality holds everywhere when both $M_{1}$ and $M_{2}$ are hyperquadrics of the same signature $\ell$. Enlightened by this result, the following conjecture concerning the CR transversality was asked by Baouendi and the first author in the year of 2005:

Conjecture (Baounedi-Huang, 2005): Let $M_{1} \subset \mathbf{C}^{n}$ and $M_{2} \subset \mathbf{C}^{N}$ be two (connected) Levi non-degenerate real analytic hypersurfaces with the same signature $0<\ell \leq \frac{n-1}{2}$. Here $3 \leq n<N$. Let $F$ be a holomorphic map defined in a neighborhood $U$ of $M_{1}$, sending $M_{1}$ into $M_{2}$. Then either $F$ is a local $C R$ embedding from $M_{1}$ into $M_{2}$ or $F$ is totally degenerate in the sense that it maps a neighborhood $U$ of $M_{1}$ in $\mathbf{C}^{n}$ into $M_{2}$.

We point out that, for the $M_{1}$ and $M_{2}$ given in the conjecture, the fact that $F$ is $\mathrm{CR}$ transversal at $p$ is equivalent to the fact that $F$ is a $\mathrm{CR}$ embedding from a neighborhood of $p$ in $M_{1}$ into $M_{2}$. Along these lines, in a recent paper of the authors [HZ2], by developing a new technique, we showed the CR transversality holds when $M_{2}=H_{\ell}^{n+1}$ and the point under study is not CR umbilical in the sense of Chern-Moser. Recall that a hyperquadric $H_{\ell}^{n}$ of signature $\ell$ in $\mathbf{C}^{n}$ is defined by

$$
H_{\ell}^{n}:=\left\{(z, w) \in \mathbf{C}^{n-1} \times \mathbf{C}: \mathfrak{I} w=|z|_{\ell}^{2}\right\},
$$

where for any $n$-tuples $a$ and $b,\langle a, \bar{b}\rangle_{\ell}:=-\sum_{j=1}^{\ell} a_{j} \bar{b}_{j}+\sum_{j=\ell+1}^{n} a_{j} \bar{b}_{j}$ and $|a|_{\ell}^{2}=\langle a, \bar{a}\rangle_{\ell}$.

In this paper, combining a quantitative version of a very useful lemma due to the first author with the tools developed in [HZ2], we are able to drop the geometric assumption of the umbilicality and relax the codimension-one restriction in [HZ2]. The generalization of the above mentioned lemma in $[\mathrm{Hu}]$ will be addressed in detail in section 3 .

We next state our main theorems: 
Theorem 1. Let $M_{\ell}$ be a smooth Levi non-degenerate hypersurface of signature $\ell$ in $\mathbf{C}^{n}$ with $n \geq 3$ and $0 \in M_{\ell}$. (We assume $0<\ell \leq \frac{n-1}{2}$.) Suppose that $F$ is a holomorphic map in a small neighborhood $U$ of $0 \in \mathbf{C}^{n}$ such that

$$
F\left(M_{\ell} \cap U\right) \subset H_{\ell}^{N}
$$

with $N-n<\frac{n-1}{2}$. If $F(U) \not \subset H_{\ell}^{N}$, then $F$ is $C R$ transversal to $M_{\ell}$ at 0 , or equivalently, $F$ is a CR embedding from a small neighborhood of $0 \in M_{\ell}$ into $H_{\ell}^{N}$.

Theorem 2. Let $M_{\ell}$ be a germ of a smooth Levi non-degenerate hypersurface at 0 of signature $\ell$ in $\mathbf{C}^{n}, n \geq 3$. (We assume $0<\ell \leq \frac{n-1}{2}$.) Suppose that there exists a holomorphic map $F$ in a neighborhood $U$ of 0 in $\mathbf{C}^{n}$ sending $M_{\ell}$ into $H_{\ell}^{N}$ but $F(U) \not \subset H_{\ell}^{N}, N<2 n-1$. Then $M_{\ell}$ is $C R$ embeddable into $H_{\ell}^{N}$ near 0 . Equivalently, there exists a holomorphic map $\tilde{F}: M_{\ell} \rightarrow H_{\ell}^{N}$ near 0 , which is CR transversal to $M_{\ell}$ at 0 .

The idea of the proof is based on a re-scaling technique that was initially introduced in [HZ2]. With the aid of a quantitative lemma of the first author in [Hu], we generate a formal CR transversal map which, by a result of Meylan-Mir-Zaitsev proved in [MMZ], is necessarily convergent. Finally, using a rigidity result in [EHZ], $F$ differs from the CR transversal map only by an automorphisms of the target and hence it is CR transversal as well.

The outline of the paper is as follows. In section 2, the notations and a normalization procedure of Baouendi-Huang is revisited. A modified lemma in [Hu] is discussed and proved in section 3. Section 4 is devoted to the proof of the main theorem.

\section{Notations and a normalization procedure}

Let $M_{\ell}$ be a germ at 0 of a smooth Levi non-degenerate hypersurface of signature $\ell$ in $\mathbf{C}^{n}$. After a holomorphic change of coordinates, $M_{\ell}$ near the origin can be expressed as follows.

$$
M_{\ell}=\left\{(z, w) \in \mathbf{C}^{n-1} \times \mathbf{C}: \mathfrak{I} w=|z|_{\ell}^{2}-\frac{1}{4} \mathscr{S}(z)+o(4)\right\} .
$$

Here $\mathscr{S}(z):=\sum_{1 \leq \alpha, \beta, \gamma, \delta \leq n} s_{\alpha \bar{\beta} \gamma \bar{\delta}} z_{\alpha} \bar{z}_{\beta} z_{\gamma} \bar{z}_{\delta}$ is a homogeneous polynomial of bi-degree $(2,2)$, called the Chern-Moser-Weyl curvature function of $M_{\ell}$ at 0 . See [CM] for more details. In what follows, we always assume that $\ell \leq(n-1) / 2$. Hence $\ell$ is a holomorphic invariant.

As in [CM], assign the weighted degree 1 to variable $z$ and 2 to variable $w$. Given a holomorphic function $h$, denote by $h^{(k)}$ the terms of weighted degree $k$, and by 
$h^{(\mu, v)}$ the terms of degree $\mu$ in $z$ variable and of degree $v$ in $w$ variable in the power series expansion of $h$ at 0 . For each integer $k \geq 0$, we write $o(k)$ for terms of degree larger than $k$, and $o_{w t}(k)$ for terms of weighted degree larger than $k$. To simplify our notation, we also preassign the coefficient of $h$ with negative degrees to be 0 .

Let $\tilde{M}_{\ell}$ be a germ at 0 of another smooth Levi-nondegenerate hypersurface in $\mathbf{C}^{N}$ of signature $\ell$ given by

$$
\tilde{M}_{\ell}=\left\{(\tilde{z}, \tilde{w}) \in \mathbf{C}^{N-1} \times \mathbf{C}: \mathfrak{I} \tilde{w}=|\tilde{z}|_{\ell}^{2}-\frac{1}{4} \tilde{\mathscr{S}}(\tilde{z})+o(4)\right\} .
$$

Here $\tilde{\mathscr{S}}$ is the corresponding Chern-Moser curvature tensor function of $\tilde{M}_{\ell}$ at 0 .

Let $F$ be a smooth CR map sending $\left(M_{\ell}, 0\right)$ into $\left(\tilde{M}_{\ell}, 0\right)$. Write

$$
F:=(\tilde{f}, g)=(f, \phi, g)
$$

with $f=\left(f_{1}, \ldots, f_{n-1}\right)$ and $\phi=\left(\phi_{1}, \ldots, \phi_{N-n}\right)$ being components of $F$. Assume that $F$ is $\mathrm{CR}$ transversal at 0 . Then, following a normalization procedure as in [§2, $\mathrm{BH}]$, we have

$$
\begin{gathered}
\tilde{z}=\left(f_{1}(z, w), \ldots, f_{n-1}(z, w), \phi_{1}(z, w), \ldots, \phi_{N-n}(z, w)\right)=\lambda z U+\mathbf{a} w+O\left(|(z, w)|^{2}\right) \\
\tilde{w}=g(z, w)=\sigma \lambda^{2} w+O\left(|(z, w)|^{2}\right) .
\end{gathered}
$$

Here $U$ can be extended to an $(N-1) \times(N-1)$ matrix $\tilde{U} \in S U(N-1, \ell)$ (namely $\langle X \tilde{U}, Y \bar{U}\rangle_{\ell}=\langle X, Y\rangle_{\ell}$ for any $\left.X, Y \in \mathbf{C}^{N-1}\right), \mathbf{a} \in \mathbf{C}^{N-1}$ and $\lambda>0, \sigma= \pm 1$ with $\sigma=1$ for $\ell<\frac{n-1}{2}$. When $\sigma=-1$, by considering $F \circ \tau_{n-1 / 2}$ instead of $F$, where $\tau_{\frac{n-1}{2}}\left(z_{1}, \ldots, z_{\frac{n-1}{2}}, z_{\frac{n-1}{2}+1}, \ldots, z_{n-1}, w\right)=\left(z_{\frac{n-1}{2}+1}, \ldots, z_{n-1}, z_{1}, \ldots, z_{\frac{n-1}{2}},-w\right)$, we can make $\sigma=1$. Hence, we will assume in what follows that $\sigma=1$. Moreover, as in [HZ], $F$ can be normalized as follows:

Proposition 1. ([(HZ]) Let $M_{\ell}$ and $\tilde{M}_{\ell}$ be defined by (17) and (2), respectively, and let $F$ be a smooth CR map sending $M_{\ell}$ into $\tilde{M}_{\ell}$ given by (3) and (4) with $\sigma=1$. Then after composing $F$ from the left by some automorphism $T \in A$ Aut $t_{0}\left(H_{\ell}^{N}\right)$ preserving the origin, the following holds:

$$
F^{\sharp}=\left(f^{\sharp}, \phi^{\sharp}, g^{\sharp}\right):=T \circ F,
$$

with

$$
\begin{gathered}
f^{\sharp}(z, w)=z+\frac{i}{2} a^{(1,0)}(z) w+o_{w t}(3), \\
\phi^{\sharp}(z, w)=\phi^{(2,0)}(z)+o_{w t}(2), \\
g^{\sharp}(z, w)=w+o_{w t}(4),
\end{gathered}
$$

and

$$
\left\langle a^{(1,0)}(z), \bar{z}\right\rangle_{\ell}|z|_{\ell}^{2}=\left|\phi^{(2,0)}(z)\right|^{2}+\frac{1}{4}\left(\mathscr{S}(z)-\lambda^{-2} \tilde{\mathscr{S}}(\lambda(z, 0) \widetilde{U})\right) .
$$

In particular, the automorphism $T$ is given by 


$$
T(\tilde{z}, \tilde{w})=\frac{\left(\lambda^{-1}\left(\tilde{z}-\lambda^{-2} \mathbf{a} \tilde{w}\right) \tilde{U}^{-1}, \lambda^{-2} \tilde{w}\right)}{q(\tilde{z}, \tilde{w})}
$$

with $r_{0}=\frac{1}{2} \Re\left\{g_{w w}^{\prime \prime}(0)\right\}, q(\tilde{z}, \tilde{w})=1+2 i\left\langle\tilde{z}, \lambda^{-2} \overline{\mathbf{a}}\right\rangle_{\ell}+\lambda^{-4}\left(r_{0}-i|\mathbf{a}|_{\ell}^{2}\right) \tilde{w}$. Moreover, $F^{\sharp}$ sends $M_{\ell}$ into $\tilde{M}^{\sharp}:=T\left(\tilde{M}_{\ell}\right)$ given by

$$
\tilde{M}^{\sharp}=\left\{\left(\tilde{z}^{\sharp}, \tilde{w}^{\sharp}\right) \in \mathbf{C}^{N+1}: \mathfrak{I} \tilde{w}^{\sharp}=\left|\tilde{z}^{\sharp}\right|_{\ell}^{2}+\frac{1}{4} \tilde{S}^{\sharp}\left(\tilde{z}^{\sharp}\right)+o(4)\right\}
$$

with $\tilde{\mathscr{S}}^{\sharp}\left(z^{\sharp}\right)=\lambda^{-2} \tilde{\mathscr{S}}\left(\lambda z^{\sharp} \tilde{U}\right)$.

\section{A quantitative version of a basic lemma}

In this section, some simple preparation facts will be given without proof at first. In the second part of the section, we will discuss a quantitative version of a lemma obtained in $[\mathrm{Hu}]$, which played crucial role for us to get the convergence in our rescaling argument.

Given a polynomial $\phi$, define $\|\phi\|$ to be the maximum modulus of all the coefficients in $\phi$. For a given vector-valued polynomial $\phi=\left(\phi_{1}, \ldots, \phi_{s}\right),\|\phi\|:=$ $\max _{1 \leq j \leq s}\left\|\phi_{j}\right\|$. We first refer to a lemma in [HZ2] without proof.

Lemma 1. HZ2 (1). Let $X(z, \bar{z})$ and $Y(z, \bar{z})$ be two polynomials such that $X(z, \bar{z})=$ $Y(z, \bar{z})|z|_{\ell}^{2}$. Then $\|Y\|$ is bounded by a constant depending only on $\|X\|$ and the degree of $X$.

(2). Let $h(z)$ be a homogeneous holomorphic polynomial of degree $d$ in $z \in \mathbf{C}^{n}$. If $|h(z)| \leq c|z|^{d}$ on $\left\{|z|_{\ell}^{2}=0\right\}$, then $\|h\| \leq C$ for some $C$ depending only on $c$ and $d$.

In various rigidity problems concerning $\mathrm{CR}$ immersions, the following lemma in [Hu] plays an essential role in deriving key identities to eventually conclude uniqueness:

Lemma 2. $\left[\mathrm{Hu}\right.$ Let $\left\{\phi_{j}\right\}_{j=1}^{n-1}$ and $\left\{\psi_{j}\right\}_{j=1}^{n-1}$ be two families of holomorphic functions in $\mathbf{C}^{n}$. Let $B(z, \xi)$ be a real-analytic function in $(z, \xi)$. Suppose that

$$
\sum_{j=1}^{n-1} \phi_{j}(z) \psi_{j}(\xi)=B(z, \xi)\langle z, \xi\rangle_{\ell}
$$

Then $B(z, \xi)=\sum_{j=1}^{n-1} \phi_{j}(z) \psi_{j}(\xi)=0$.

We find a quantitative version of the above lemma serves our purpose under this context perfectly well. 
Lemma 3. Let $\left\{\phi_{j}\right\}_{j=1}^{n-1}$ and $\left\{\psi_{j}\right\}_{j=1}^{n-1}$ be two families of holomorphic polynomials of degree $k$ and $m$ in $\mathbf{C}^{n}$, respectively. Let $H(z, \xi), B(z, \xi)$ be two polynomials in $(z, \xi)$. Suppose that

$$
\sum_{j=1}^{n-1} \phi_{j}(z) \psi_{j}(\xi)=H(z, \xi)+B(z, \xi)\langle z, \xi\rangle_{\ell}
$$

and $\|H\| \leq C$. Then $\|B\| \leq \tilde{C}$ and $\left\|\sum_{j=1}^{n-1} \phi_{j}(z) \psi_{j}(\xi)\right\| \leq \tilde{C}$ with $\tilde{C}$ dependent only on $(C, k, m, n)$.

The proof of the lemma is based on the following algorithm together with Lemma 2 First, let us formulate the algorithm procedure so as to re-adjust two families $\left\{\phi_{j}\right\}_{j=1}^{n-1}$ and $\left\{\psi_{j}\right\}_{j=1}^{n-1}$ in Lemma 3 .

Lemma 4. Let $\phi:=\left\{\phi_{j}\right\}_{j=1}^{s}$ and $\psi:=\left\{\psi_{j}\right\}_{j=1}^{s}$ be two families of holomorphic polynomials of degree $k$ and $m$ in $\mathbf{C}^{n}$, respectively. There exist two families $\tilde{\phi}:=$ $\left\{\tilde{\phi}_{j}\right\}_{j=1}^{s}$ and $\tilde{\psi}:=\left\{\tilde{\psi}_{j}\right\}_{j=1}^{s}$ of holomorphic polynomials of degree $k$ and $m$ in $\mathbf{C}^{n}$, respectively, such that

$$
\sum_{j=1}^{s} \phi_{j}(z) \psi_{j}(\xi)=\sum_{j=1}^{s} \tilde{\phi}_{j}(z) \tilde{\psi}_{j}(\xi)
$$

and

$$
\|\tilde{\phi}\| \leq 1, C\|\tilde{\psi}\| \leq\left\|\sum_{j=1}^{s} \tilde{\phi}_{j}(z) \tilde{\psi}_{j}(\xi)\right\| \leq s\|\tilde{\psi}\|
$$

for some positive constant $C$ dependent only on s.

Proof of Lemma 4; Without loss of generality, assume $\left\|\phi_{j}\right\| \neq 0$ for all $1 \leq j \leq s$ and $\left\{\phi_{j}\right\}_{j=1}^{s}$ are linearly independent. Moreover, by replacing $\phi_{j}$ and $\psi_{j}$ by $\frac{\phi_{j}}{\left\|\phi_{j}\right\|}$ and $\left\|\phi_{j}\right\| \psi_{j}$, respectively, one can assume that $\left\|\phi_{j}\right\|=1$ for all $1 \leq j \leq s$. Denote by $\left\{e_{l}\right\}_{l=1}^{d(k)}$ a basis of unit monomials to span the polynomial spaces of degree $k$ and write $\phi_{j}=\sum_{1 \leq l \leq d(k)} D_{j}^{l} e_{l}, 1 \leq j \leq s$. Here $d(k)$ is the dimension of polynomial spaces of degree $k$. Hence $\left\|\phi_{j}\right\|=\max _{1 \leq l \leq d(k)} D_{j}^{l}$ for each $1 \leq j \leq s$. Arranging the order of $\left\{e_{l}\right\}$ if necessary, we can make $D_{1}^{1}=1$ and $\left|D_{1}^{l}\right| \leq 1$.

Step 1: Let ${ }^{1} \phi_{1}:=\phi_{1},{ }^{1} \phi_{j}:=\phi_{j}-D_{j}^{1} \cdot \phi_{1}, 2 \leq j \leq s$. Then in terms of the basis representation ${ }^{1} \phi_{j}:={ }^{1} D_{j}^{l} \cdot e_{l}$, one has

$$
\begin{gathered}
{ }^{1} D_{1}^{1}=1,\left|{ }^{1} D_{1}^{l}\right| \leq 1,2 \leq l \leq d(k) ; \\
{ }^{1} D_{j}^{1}=0,\left|{ }^{1} D_{j}^{l}\right| \leq 2,2 \leq j \leq s, 2 \leq l \leq d(k) .
\end{gathered}
$$

Moreover, letting ${ }^{1} \psi_{1}:=\psi_{1}+\sum_{j=2}^{s} D_{j}^{1} \cdot \psi_{j},{ }^{1} \psi_{j}:=\psi_{j}, 2 \leq j \leq s$, then 


$$
\sum_{j=1}^{s} \phi_{j}(z) \psi_{j}(\xi)=\sum_{j=1}^{s}{ }^{1} \phi_{j}(z) \cdot{ }^{1} \psi_{j}(\xi) .
$$

Step 2: Normalize ${ }^{1} \phi_{j}, 2 \leq j \leq s$ by replacing ${ }^{1} \phi_{j},{ }^{1} \psi_{j}$ by $\frac{{ }^{1} \phi_{j}}{\left\|^{1} \phi_{j}\right\|}$ and $\left\|{ }^{1} \phi_{j}\right\| \cdot{ }^{1} \psi_{j}$, respectively. By abuse of notation, we still denote them by ${ }^{1} \phi_{j},{ }^{1} \psi_{j}$ and the representation matrix under the basis $\left\{e_{l}\right\}$ by $\left\{{ }^{1} D_{j}^{l}\right\}$. Moreover, since $\left\{\phi_{j}\right\}_{j=1}^{s}$ are linearly independent, by rearranging the order of $\left\{e_{l}\right\}_{l=2}^{d(k)}$ if necessary, we have (7) holds with

$$
\begin{gathered}
{ }^{1} D_{1}^{1}=1,\left|{ }^{1} D_{1}^{l}\right| \leq 1,2 \leq l \leq d(k) ; \\
{ }^{1} D_{2}^{1}=0,{ }^{1} D_{2}^{2}=1,\left|{ }^{1} D_{2}^{l}\right| \leq 1,3 \leq l \leq d(k) ; \\
{ }^{1} D_{j}^{1}=0,\left|{ }^{1} D_{j}^{l}\right| \leq 1,3 \leq j \leq s, 2 \leq l \leq d(k)
\end{gathered}
$$

and for each $1 \leq j \leq s$,

$$
\max _{1 \leq l \leq d(k)}{ }^{1} D_{j}^{l}=1 .
$$

Step 3: Let ${ }^{2} \phi_{2}={ }^{1} \phi_{2},{ }^{2} \phi_{j}:={ }^{1} \phi_{j}-{ }^{1} D_{j}^{2} \cdot{ }^{1} \phi_{2}$ for $1 \leq j \leq s, j \neq 2$. Then in terms of the basis representation ${ }^{2} \phi_{j}:={ }^{2} D_{j}^{l} \cdot e_{l}$, we deduce

$$
\begin{gathered}
{ }^{2} D_{1}^{1}=1,{ }^{2} D_{1}^{2}=0,\left|{ }^{2} D_{1}^{l}\right| \leq 2,3 \leq l \leq d(k) ; \\
{ }^{2} D_{2}^{1}=0,{ }^{2} D_{2}^{2}=1,\left|{ }^{2} D_{2}^{l}\right| \leq 1,3 \leq l \leq d(k) ; \\
{ }^{2} D_{j}^{1}=0,{ }^{2} D_{j}^{2}=0,\left|{ }^{2} D_{j}^{l}\right| \leq 2,3 \leq j \leq s, 3 \leq l \leq d(k) .
\end{gathered}
$$

Moreover, letting ${ }^{2} \psi_{2}:={ }^{1} \psi_{2}+\sum_{j \neq 2}{ }^{1} D_{j}^{2} \cdot{ }^{1} \psi_{j},{ }^{2} \psi_{j}:={ }^{1} \psi_{j}, 1 \leq j \leq s$ with $j \neq 2$, then

$$
\sum_{j=1}^{s} \phi_{j}(z) \psi_{j}(\xi)=\sum_{j=1}^{s}{ }^{2} \phi_{j}(z) \cdot{ }^{2} \psi_{j}(\xi)
$$

Step 4: Normalize ${ }^{2} \phi_{j}, 1 \leq j \leq s, j \neq 2$ by replacing ${ }^{2} \phi_{j},{ }^{2} \psi_{j}$ by $\frac{{ }^{2} \phi_{j}}{\left\|^{2} \phi_{j}\right\|}$ and $\left\|^{2} \phi_{j}\right\|$. ${ }^{2} \psi_{j}$, respectively. As before, we still denote them by ${ }^{2} \phi_{j},{ }^{2} \psi_{j}$ and the representation matrix under the basis $\left\{e_{l}\right\}$ by $\left\{{ }^{2} D_{j}^{l}\right\}$. Furthermore, 8 holds with

$$
\begin{gathered}
1 \geq{ }^{2} D_{1}^{1} \geq \frac{1}{2},{ }^{2} D_{1}^{2}=0,\left|{ }^{2} D_{1}^{l}\right| \leq 1,3 \leq l \leq d(k) ; \\
{ }^{2} D_{2}^{1}=0,{ }^{2} D_{2}^{2}=1,\left|{ }^{2} D_{2}^{l}\right| \leq 1,3 \leq l \leq d(k) ; \\
{ }^{2} D_{j}^{1}=0,{ }^{2} D_{j}^{2}=0,\left|{ }^{2} D_{j}^{l}\right| \leq 1,3 \leq j \leq s, \quad 3 \leq l \leq d(k)
\end{gathered}
$$

and for each $1 \leq j \leq s$,

$$
\max _{1 \leq l \leq d(k)}{ }^{2} D_{j}^{l}=1 .
$$

Step 5: Continue the above process until we get new families $\left\{{ }^{s} \phi_{j}\right\}_{j=1}^{s},\left\{{ }^{s} \psi_{j}\right\}_{j=1}^{s}$ such that under the basis representation, ${ }^{s} \phi_{j}:={ }^{s} D_{j}^{l} \cdot e_{l}$ with 


$$
{ }^{s} D=\left[\begin{array}{cccccccc}
{ }^{s} D_{1}^{1} & 0 & 0 & \ldots & 0 & { }^{s} D_{1}^{s+1} & \ldots & { }^{s} D_{1}^{d(k)} \\
0 & { }^{s} D_{2}^{2} & 0 & \ldots & 0 & { }^{s} D_{2}^{s+1} & \ldots & { }^{s} D_{2}^{d(k)} \\
0 & 0 & { }^{s} D_{3}^{3} & \ldots & 0 & { }^{s} D_{2}^{s+1} & \ldots & { }^{s} D_{3}^{d(k)} \\
& & & & \ldots & & & \\
& & & & \ldots & & & \\
& & & & \ldots & & & \\
0 & 0 & 0 & \ldots & { }^{s} D_{s}^{s} & { }^{s} D_{s}^{s+1} & \ldots & { }^{s} D_{s}^{d(k)}
\end{array}\right]
$$

where

$$
1 \geq{ }^{s} D_{j}^{j} \geq \frac{1}{2^{s-j}}, 1 \leq j \leq s-1 ;{ }^{s} D_{s}^{s}=1 ;
$$

and for each $1 \leq j \leq s$,

$$
\max _{1 \leq l \leq d(k)}{ }^{s} D_{j}^{l}=1
$$

Moreover,

$$
\sum_{j=1}^{s} \phi_{j}(z) \psi_{j}(\xi)=\sum_{j=1}^{s}{ }^{s} \phi_{j}(z) \cdot{ }^{s} \psi_{j}(\xi)
$$

Let $\tilde{\phi}_{j}:={ }^{s} \phi_{j}, \tilde{\psi}_{j}:={ }^{s} \psi_{j}, 1 \leq j \leq s$. Then from the construction, for $1 \leq j \leq s$, $\left\|\tilde{\phi}_{j}\right\|=1$ with $\sum_{j=1}^{s} \phi_{j}(z) \psi_{j}(\xi)=\sum_{j=1}^{s} \tilde{\phi}_{j}(z) \tilde{\psi}_{j}(\xi)$. Hence

$$
\left\|\sum_{j=1}^{s} \phi_{j}(z) \psi_{j}(\xi)\right\| \leq \sum_{j=1}^{s}\left\|\tilde{\phi}_{j}\right\|\left\|\tilde{\psi}_{j}\right\| \leq s\|\tilde{\psi}\| .
$$

Furthermore, since ${ }^{s} D_{j}^{j} \geq \frac{1}{2^{s-j}}$ when $1 \leq j \leq s$,

$$
\left\|\sum_{j=1}^{s} \phi_{j}(z) \psi_{j}(\xi)\right\| \geq \max _{1 \leq j \leq s}{ }^{s} D_{j}^{j} \cdot\left\|\tilde{\psi}_{j}\right\| \geq \frac{1}{2^{s-1}}\|\tilde{\psi}\| .
$$

The proof of Lemma 4 is therefore complete.

Proof of Lemma 3: Assume by contradiction that there exist families of $\left\{\phi^{\lambda}\right\}$ and $\left\{\psi^{\lambda}\right\}$, such that

$$
\sum_{j=1}^{n-1} \phi_{j}^{\lambda}(z) \psi_{j}^{\lambda}(\xi)=H^{\lambda}(z, \xi)+B^{\lambda}(z, \xi)\langle z, \xi\rangle_{\ell}
$$

with $\left\|H^{\lambda}\right\| \leq C$ while $\left\|\sum_{j=1}^{n-1} \phi_{j}^{\lambda}(z) \psi_{j}^{\lambda}(\xi)\right\|=\lambda \rightarrow \infty$. Applying Lemma4 to $\phi^{\lambda}$ and $\psi^{\lambda}$ if necessary, we can further assume that $\phi^{\lambda}$ and $\psi^{\lambda}$ satisfy 


$$
\left\|\phi^{\lambda}\right\| \leq 1, \quad C\left\|\psi^{\lambda}\right\| \leq\left\|\sum_{j=1}^{n-1} \phi_{j}^{\lambda}(z) \psi_{j}^{\lambda}(\xi)\right\|=\lambda \leq(n-1)\left\|\psi^{\lambda}\right\| .
$$

In special, for each $1 \leq j \leq n-1$,

$$
\left\|\phi_{j}^{\lambda}\right\| \leq 1, \frac{1}{n-1} \leq\left\|\frac{\psi_{j}^{\lambda}}{\lambda}\right\| \leq \frac{1}{C}
$$

Dividing both sides of (9) by $\lambda$, then one obtains for some polynomial $\tilde{B}^{\lambda}$ that

$$
\sum_{j=1}^{n-1} \phi_{j}^{\lambda}(z) \frac{\psi_{j}^{\lambda}(\xi)}{\lambda}=\frac{H^{\lambda}(z, \xi)}{\lambda}+\tilde{B}^{\lambda}(z, \xi)\langle z, \xi\rangle_{\ell} .
$$

Since $\phi^{\lambda}$ and $\psi^{\lambda}$ satisfy (10), we deduce after passing to a subsequence that $\phi^{\lambda}$ and $\frac{\psi^{\lambda}}{\lambda}$ converges, say, to polynomials $\phi^{\infty}$ and $\psi^{\infty}$. Moreover, the same inequalities in (10) pass onto $\phi^{\infty}$ and $\psi^{\infty}$ without change, i.e., $\left\|\phi^{\infty}\right\| \leq 1, \frac{1}{n-1} \leq\left\|\psi^{\infty}\right\| \leq C$ and $C\left\|\psi^{\infty}\right\| \leq\left\|\sum_{j=1}^{n-1} \phi_{j}^{\infty}(z) \psi_{j}^{\infty}(\xi)\right\| \leq(n-1)\left\|\psi^{\infty}\right\|$.

On the other hand, from (11) and Lemma 11, after passing $\lambda \rightarrow \infty$, there exists some polynomial $B^{\infty}$ such that

$$
\sum_{j=1}^{n-1} \phi_{j}^{\infty}(z) \psi_{j}^{\infty}(\xi)=B^{\infty}(z, \xi)\langle z, \xi\rangle_{\ell} .
$$

According to Lemma2 it immediately gives that

$$
\sum_{j=1}^{n-1} \phi_{j}^{\infty}(z) \psi_{j}^{\infty}(\xi)=0
$$

This however contradicts with the fact that $\left\|\sum_{j=1}^{n-1} \phi_{j}^{\infty}(z) \psi_{j}^{\infty}(\xi)\right\| \geq C\left\|\psi^{\infty}\right\| \geq \frac{C}{n-1}$.

Therefore, there exists some $\tilde{C}$ dependent only on $(C, k, m, n)$ such that $\left\|\sum_{j=1}^{n-1} \phi_{j}(z) \psi_{j}(\xi)\right\|$ $\leq \tilde{C}$ and hence $\|B\| \leq \tilde{C}$ because of Lemma1,

With a routine induction process, Lemmas 1 and 3 combined together can be used to show the following:

Lemma 5. Let $\left\{\phi_{j r}\right\}_{j=1}^{n-1}$ and $\left\{\psi_{j r}\right\}_{j=1}^{n-1}$ be two families of holomorphic polynomials in $\mathbf{C}^{n}, 1 \leq r \leq m$. Let $H(z, \xi), B(z, \xi)$ be two polynomials in $(z, \xi)$. Suppose that

$$
\sum_{r=1}^{m}\left(\sum_{j=1}^{n-1} \phi_{j r}(z) \psi_{j r}(\xi)\right)\langle z, \xi\rangle_{\ell}^{r}=H(z, \xi)+B(z, \xi)\langle z, \xi\rangle_{\ell}^{m+1}
$$


and $\|H\| \leq C$. Then $\|B\| \leq \tilde{C}$ and $\left\|\sum_{j=1}^{n-1} \phi_{j r}(z) \psi_{j r}(\xi)\right\| \leq \tilde{C}$ for all $1 \leq r \leq m$ with $\tilde{C}$ dependent only on $(C, n, m)$ and the degrees of $\phi_{j r}, \psi_{j r}$ for all $1 \leq r \leq m$.

\section{Proof of the main theorems}

The proof of the main theorems is motivated by the ideas in [HZ2] and [EHZ]. Assume $F$ is not CR transversal to $M_{\ell}$ at 0 and $F(U) \not \subset H_{\ell}^{N}$. Assume also $N-n<$ $n-1$ for the moment.

By a result of [BER2], the set of points where the CR transversality holds for such an $F$ forms an open dense subset in $M_{\ell}$. Choose a sequence $\left\{p_{j}\right\} \in M_{\ell}$ such that $p_{j} \rightarrow 0$ and $F$ is CR transversal at each $p_{j}$ with $j \geq 1$. Write $q_{j}:=F\left(p_{j}\right)$. Now for each $j$, applying the normalization process to $F$ at $p_{j}$ as in section 2, we obtain $F_{p_{j}}^{\sharp}$ in the following form:

$$
F_{p_{j}}^{\sharp}=\left(f_{p_{j}}^{\sharp}, \phi_{p_{j}}^{\sharp}, g_{p_{j}}^{\sharp}\right)=\left(f_{1_{p_{j}}}^{\sharp}, \ldots, f_{n_{p_{j}}}^{\sharp}, \phi_{p_{j}}^{\sharp}, g_{p_{j}}^{\sharp}\right):=T_{p_{j}} \circ \tau_{F\left(p_{j}\right)} \circ F \circ \sigma_{p_{j}},
$$

where

$$
\begin{gathered}
f_{p_{j}}^{\sharp}(z, w)=z+\frac{i}{2} a_{p_{j}}^{(1,0)}(z) w+o_{w t}(3), \\
\phi_{p_{j}}^{\sharp}(z, w)=\phi_{p_{j}}^{(2,0)}(z)+o_{w t}(2), \\
g_{p_{j}}^{\sharp}(z, w)=w+o_{w t}(4),
\end{gathered}
$$

with the following CR Gauss-Codazzi equation

$$
\left\langle a_{p_{j}}^{(1,0)}(z), \bar{z}\right\rangle_{\ell}|z|_{\ell}^{2}=\left|\phi_{p_{j}}^{(2,0)}(z)\right|^{2}+\frac{1}{4} \mathscr{S}_{p_{j}}(z)
$$

Here $\tau_{F\left(p_{j}\right)}$ is the translation map of $H_{\ell}^{N}$ sending $F\left(p_{j}\right)$ to $0, \sigma_{p_{j}}$ is a biholomorphic map sending 0 to $p_{j}$ such that $\sigma_{p_{j}}^{-1}\left(M_{\ell}\right)$ is normalized up to the 4 th order, and $\mathscr{S}_{p_{j}}$ is the resulting Chern-Moser-Weyl curvature function of $M_{\ell}$ at $p_{j}$. Note $\sigma_{p_{j}}$ depends smoothly on $p_{j}$. Since $F$ is not CR transversal at $0, \lim _{j \rightarrow \infty} \lambda_{p_{j}}=0$ with $\lambda_{p_{j}}$ defined in (4) for the map $\tau_{F\left(p_{j}\right)} \circ F \circ \sigma_{p_{j}}$. By construction, at each point $p_{j}, F_{p_{j}}^{\sharp}$ sends $\sigma_{p_{j}}^{-1}\left(M_{\ell}\right)$ into $H_{\ell}^{N}$. We then have for $(z, u) \approx 0$,

$$
\begin{aligned}
-\mathfrak{I} g_{p_{j}}^{\sharp}\left(z, u+i\left(|z|_{\ell}^{2}\right.\right. & \left.\left.+o_{w t}(3)\right)\right)+\left|f_{p_{j}}^{\sharp}\left(z, u+i\left(|z|_{\ell}^{2}+o_{w t}(3)\right)\right)\right|_{\ell}^{2}+ \\
& +\left|\phi_{p_{j}}^{\sharp}\left(z, u+i\left(|z|_{\ell}^{2}+o_{w t}(3)\right)\right)\right|^{2}=0,
\end{aligned}
$$

Here $\left(z, u+i\left(|z|_{\ell}^{2}+o_{w t}(3)\right)\right)$ is a local parametrization of $\sigma_{p_{j}}^{-1}\left(M_{\ell}\right)$ near 0 . Due to the smooth dependence of $\sigma_{p_{j}}$ with respect to $p_{j}$, the error term $o_{w t}(3)$ depends 
smoothly on $p_{j}$. With an abuse of notation, we shall suppress $\sharp$ and the subindex $j$ of $p$ for the map in (14).

Given any positive integer $k \geq 2$, collect terms of weighted degree $k$ in the power series expansion of (14). We have:

$$
\begin{gathered}
\quad \mathfrak{J} g_{p}^{(k)}(z, w)-2 \mathfrak{R}\left\langle f_{p}^{(k-1)}(z, w), \bar{z}\right\rangle_{\ell}=\left(\left|\phi_{p}(z, w)\right|^{2}\right)^{(k)} \\
+\quad H\left(\left.g_{p}^{(r)}\right|_{0 \leq r \leq k-1},\left.f_{p}^{(r)}\right|_{0 \leq r \leq k-2},\left.\left(\left|\phi_{p}\right|^{2}\right)^{(r)}\right|_{0 \leq r \leq k-1}\right)
\end{gathered}
$$

on $w=u+i|z|_{\ell}^{2}$. Here $H$ is a certain bounded polynomial on all its variables. From now on and in what follows, we use $C$ in general to represent constants independent of $p$, and use $H(\cdot, \cdot)$ in general to represent polynomials whose norm is bounded by C. $C$ and $H$ may be different in different contexts.

Lemma 6. Assume that $N-n<n-1$. For $F_{p}$ constructed as above and for each $k$, $\left\|F_{p}^{(k)}\right\| \leq C$ with $C$ independent of $p$.

Proof of Lemma 6 According to the normalization procedure conducted in Section 2, $\left\|g_{p}^{(k)}\right\| \leq C,\left\|f_{p}^{(k-1)}\right\| \leq C,\left\|\left(\left|\phi_{p}\right|^{2}\right)^{(k)}\right\| \leq C$ automatically hold when $k \leq 4$. Indeed, $\left\|g_{p}^{(k)}\right\| \leq 1,\left\|f_{p}^{(k-2)}\right\| \leq 1,\left\|\left(\left|\phi_{p}\right|^{2}\right)^{(k-1)}\right\|=0, k \leq 4$ by (12). Moreover, since $\left\|\mathscr{S}_{p}\right\| \leq$ $C$, applying Lemmaß 3 to $(13)$, one has $\left\|f_{p}^{(3)}\right\| \leq C,\left\|\left(\left|\phi_{p}\right|^{2}\right)^{(4)}\right\| \leq C$.

Assuming by induction that $\left(\left\|g_{p}^{(j)}\right\|,\left\|f_{p}^{(j-1)}\right\|,\left\|\left(\left|\phi_{p}\right|^{2}\right)^{(j)}\right\|\right)$ are all uniformly bounded by some constant independent of $p$ for $j \leq k$, we shall show the unform boundedness of $\left(\left\|g_{p}^{(k+1)}\right\|,\left\|f_{p}^{(k)}\right\|,\left\|\left(\left|\phi_{p}\right|^{2}\right)^{(k+1)}\right\|\right)$. Complexifying (15) at level $k+1$, we obtain

$$
\begin{gathered}
g_{p}^{(k+1)}(z, w)-\bar{g}_{p}^{(k+1)}(\xi, \eta)-2 i\left\langle f_{p}^{(k)}(z, w), \xi\right\rangle_{\ell}-2 i\left\langle\bar{f}_{p}^{(k)}(\xi, \eta), z\right\rangle_{\ell} \\
=2 i\left\langle\phi_{p}(z, w), \bar{\phi}_{p}(\xi, \eta)\right\rangle^{(k+1)}+H(z, \xi, w, \eta)
\end{gathered}
$$

which holds on $w-\eta=2 i\langle z, \xi\rangle_{\ell}$.

Let $L_{j}=\frac{\partial}{\partial z_{j}}+2 i \delta_{j} \xi_{j} \frac{\partial}{\partial w}, 1 \leq j \leq n-1$ with $\delta_{j}=-1$ when $j \leq \ell$ and $\delta_{j}=1$ with $j \geq \ell+1$. Then $L_{j}$ is a holomorphic tangent vector field on $w-\eta=2 i\langle z, \xi\rangle_{\ell}$ for each $j$. Applying $L_{j}$ onto (16), we get

$$
\begin{gathered}
L_{j} g_{p}^{(k+1)}(z, w)-2 i\left\langle L_{j} f_{p}^{(k)}(z, w), \xi\right\rangle_{\ell}-2 i \bar{f}_{p, j}^{(k)}(\xi, \eta) \\
=2 i L_{j}\left\langle\phi_{p}(z, w), \bar{\phi}_{p}(\xi, \eta)\right\rangle^{(k+1)}+H(z, \xi, w, \eta)
\end{gathered}
$$

on $w-\eta=2 i\langle z, \xi\rangle_{\ell}$.

Now we expand $g_{p}^{(k+1)}, f_{p}^{(k)},\left\langle\phi_{p}(z, w), \bar{\phi}_{p}(\xi, \eta)\right\rangle^{(k+1)}$ in the following manner:

$$
\begin{aligned}
g_{p}^{(k+1)}(z, w) & =\sum_{\mu+2 v=k+1}\left(g_{p}\right)_{\mu v}(z) w^{v} ; \\
f_{p}^{(k)}(z, w) & =\sum_{\mu+2 v=k}\left(f_{p}\right)_{\mu v}(z) w^{v} ;
\end{aligned}
$$




$$
\left\langle\phi_{p}(z, w), \bar{\phi}_{p}(\xi, \eta)\right\rangle^{(k+1)}=\sum_{\mu+\gamma+2(v+\delta)=k+1}\left(A_{p}\right)_{\mu \gamma v \delta}(z, \xi) w^{v} \eta^{\delta} .
$$

Here $\left(g_{p}\right)_{\mu \nu}$ and $\left(f_{p}\right)_{\mu \nu}$ are homogeneous polynomials of degree $\mu$ in $z,\left(A_{p}\right)_{\mu \gamma v \delta}$ is a homogeneous polynomial of bi-degree $(\mu, \gamma)$ in $(z, \xi)$.

Let $w=0, \eta=-2 i\langle z, \xi\rangle_{\ell}$ in (16). Then we have

$$
\begin{gathered}
\left(g_{p}\right)_{(k+1) 0}(z)-\sum_{\mu+2 v=k+1}\left(\bar{g}_{p}\right)_{\mu v}(\xi) \eta^{v}-2 i\left\langle\left(f_{p}\right)_{k 0}(z), \xi\right\rangle_{\ell} \\
\left.-2 i \sum_{\mu+2 v=k}\left(\bar{f}_{p}\right)_{\mu v}(\xi) \eta^{v}, z\right\rangle_{\ell}=2 i \sum_{\mu+\gamma+2 \delta=k+1}\left(A_{p}\right)_{\mu \gamma 0 \delta}(z, \xi) \eta^{\delta}+H(z, \xi, \eta)(18)
\end{gathered}
$$

on $\eta=-2 i\langle z, \xi\rangle_{\ell}$.

Collect terms in $(z, \xi)$ of bi-degree $(k+1,0)$ and $(k, 1)$ in (18). By the fact that $\phi_{p}(0)=\frac{\partial \phi_{p}}{\partial z}(0)=\frac{\partial \phi_{p}}{\partial w}(0)=0$ and the definition of $\left(A_{p}\right)_{\mu \gamma v \delta}$,

$$
\left(A_{p}\right)_{k+1,0,0,0}=\left(A_{p}\right)_{k, 1,0,0}=\left(A_{p}\right)_{k-1,0,0,1}=0 .
$$

Then we have that

$$
\left\|\left(f_{p}\right)_{k 0}\right\| \leq C, \quad\left\|\left(g_{p}\right)_{(k+1) 0}\right\| \leq C .
$$

Hence for each $1 \leq j \leq n-1$,

$$
\begin{gathered}
L_{j} f_{p}^{(k)}(z, 0)=2 i \delta_{j} \xi_{j} \sum_{\mu=k-2}\left(f_{p}\right)_{\mu 1}(z)+H(z) ; \\
L_{j} g_{p}^{(k+1)}(z, 0)=2 i \delta_{j} \xi_{j} \sum_{\mu=k-1}\left(g_{p}\right)_{\mu 1}(z)+H(z) .
\end{gathered}
$$

Collecting terms in $(z, \xi)$ of bi-degree $(\alpha, \beta), \beta \geq 2$ with $\alpha+\beta=k+1$ in 18 gives

$$
\begin{gathered}
-\left(\bar{g}_{p}\right)_{\beta-\alpha, \alpha}(\xi) \eta^{\alpha}-2 i\left\langle z,\left(\bar{f}_{p}\right)_{\beta-\alpha+1, \alpha-1}(\xi) \eta^{\alpha-1}\right\rangle_{\ell} \\
=2 i \sum_{\theta=0}^{\alpha-2}\left(A_{p}\right)_{\alpha-\theta, \beta-\theta, 0, \theta}(z, \xi) \eta^{\theta}+H(z, \xi, \eta)
\end{gathered}
$$

with $\eta=-2 i\langle z, \xi\rangle_{\ell}$. Here once again we used the fact that $\phi_{p}(0)=\frac{\partial \phi_{p}}{\partial z}(0)=0$ which implies $\left(A_{p}\right)_{1,(\beta-\alpha-1), 0,(\alpha-1)}=\left(A_{p}\right)_{0, \beta-\alpha, 0, \alpha}=0$, so the summation on the right hand side runs only till $\theta=\alpha-2$. Recall from the definition of $A_{p}$, $\left(A_{p}\right)_{\mu \gamma v \delta}(z, \xi)=\sum_{j=1}^{N-n} \phi_{p, j}^{(\mu, v)}(z, 1) \bar{\phi}_{p . j}^{(\gamma, \delta)}(\xi, 1)$. Since $N-n<n-1$ by assumption, we immediately have, by applying Lemma 5 to $(21)$ and by using (19), that

$$
\left\|\left(\bar{g}_{p}\right)_{\beta-\alpha, \alpha}(\xi)\langle z, \xi\rangle_{\ell}-\left\langle z,\left(\bar{f}_{p}\right)_{\beta-\alpha+1, \alpha-1}(\xi)\right\rangle_{\ell}\right\| \leq C
$$

with $\beta \geq 2$, and

$$
\left\|\left(A_{p}\right)_{\mu \gamma 0 \delta}\right\| \leq C .
$$


Hence from the above inequality,

$$
L_{j}\left(A_{p}\right)(z, \xi, 0, \eta)=2 i \delta_{j} \xi_{j} \sum_{\mu+\gamma+2 \delta=k-1}\left(A_{p}\right)_{\mu \gamma 1 \delta}(z, \xi) \eta^{\delta}+H(z, \xi, \eta) .
$$

Letting $w=0, \eta=-2 i\langle z, \xi\rangle_{\ell}$ and then substituting (20) and (23) in (17), we have for each $1 \leq j \leq n-1$,

$$
\begin{gathered}
2 i \delta_{j} \xi_{j} \sum_{\mu=k-1}\left(g_{p}\right)_{\mu 1}(z)-2 i\left\langle 2 i \delta_{j} \xi_{j} \sum_{\mu=k-2}\left(f_{p}\right)_{\mu 1}(z), \xi\right\rangle_{\ell}-2 i \sum_{\mu+2 v=k+1}\left(\bar{f}_{p, j}\right)_{\mu v}(\xi) \eta^{v} \\
=2 i \delta_{j} \xi_{j} \sum_{\mu+\gamma+2 \delta=k-1}\left(A_{p}\right)_{\mu \gamma 1 \delta}(z, \xi) \eta^{\delta}+H(z, \xi, \eta)
\end{gathered}
$$

on $\eta=-2 i\langle z, \xi\rangle_{\ell}$. Collect terms in $(z, \xi)$ of bi-degree $(k-1,1)$ and $(k-2,2)$ in 24). Since $\left(A_{p}\right)_{k-1,0,1,0}=\left(A_{p}\right)_{k-3,0,1,1}=0$, one obtains that

$$
\begin{gathered}
\left\|\left(g_{p}\right)_{(k-1) 1}\right\| \leq C, \\
\left\|2 i \delta_{j}\left\langle\xi_{j}\left(f_{p}\right)_{(k-2) 1}(z), \xi\right\rangle_{\ell}+\left(\bar{f}_{p, j}\right)_{(4-k)(k-2)}(\xi)\left(-2 i\langle z, \xi\rangle_{\ell}\right)^{k-2}\right\| \leq C .
\end{gathered}
$$

Here we have used the convention that $h_{\mu}=0$ if $\mu$ is negative.

Moreover, collecting terms of bi-degree $(\alpha, \beta)$ in $(z, \xi)$ with $\beta \geq 3$ and $\alpha+\beta=k$ in 24], one gets for each $1 \leq j \leq n$,

$$
\begin{gathered}
\left(\bar{f}_{p, j}\right)_{(\beta-\alpha) \alpha}(\xi)\left(-2 i\langle z, \xi\rangle_{\ell}\right)^{\alpha} \\
=-\delta_{j} \xi_{j} \sum_{\theta=0}^{\alpha-1}\left(A_{p}\right)_{(\alpha-\theta)(\beta-\theta-1) 1 \theta}(z, \xi)\left(-2 i\langle z, \xi\rangle_{\ell}\right)^{\theta}+H(z, \xi) .
\end{gathered}
$$

Here we use the fact that $\left(A_{p}\right)_{0, \beta-\alpha-1,1, \alpha}=0$, so the summation on the right hand sides runs only till $\alpha-1$. Applying Lemma 5 onto the above identity as before, we obtain $\left\|\left(f_{p}\right)_{\mu v}\right\| \leq C$ for $\mu+2 v=k$ with $\mu+v \geq 3$. When $\mu+2 v=k \geq 4$ with $\mu+v \leq 2$, or equivalently, when $\mu=0, v=2$, one substitutes the fact that $\left\|\left(f_{p}\right)_{21}\right\| \leq C$ into 25] and gets $\left\|\left(f_{p}\right)_{02}\right\| \leq C$ and hence

$$
\left\|\left(f_{p}\right)_{\mu v}\right\| \leq C
$$

for $\mu+2 v=k$. Substitute (26) into 221, then

$$
\left\|\left(g_{p}\right)_{\mu v}\right\| \leq C
$$

for $\mu+2 v=k+1$ (with $\mu+v \geq 3$, which is always fulfilled when $\mu+2 v=k+1 \geq$ $5)$.

Using equation (16), we then have from (26) and (27),

$$
\left\langle\phi_{p}(z, w), \bar{\phi}_{p}(\xi, \eta)\right\rangle^{(k+1)}=H(z, \xi, w, \eta)
$$

on $w-\eta=2 i\langle z, \xi\rangle_{\ell}$.

We claim that, for arbitrary $(z, w, \xi, \eta)$, we have 


$$
\left\langle\phi_{p}(z, w), \bar{\phi}_{p}(\xi, \eta)\right\rangle^{(k+1)}=H(z, \xi, w, \eta) .
$$

Indeed, by 28, we have

$$
\sum_{\mu+\gamma+2(v+\delta)=k+1}\left(A_{p}\right)_{\mu \gamma \nu \delta}(z, \xi)\left(\eta+2 i\langle z, \xi\rangle_{\ell}\right)^{v} \eta^{\delta}=H(z, \xi, \eta)
$$

near 0. If $\left\|\left(A_{p}\right)_{\mu \gamma v \delta}\right\| \leq C$ does not hold uniformly in $p$, then there exists a smallest integer $\delta_{0}$ such that $\left\|\left(A_{p}\right)_{\mu \gamma \nu \delta_{0}}\right\| \rightarrow \infty$ as $p \rightarrow 0$ after passing to a subsequence if necessary. Moving the terms with $\delta<\delta_{0}$ to the right, we obtain

$$
\sum_{\mu+\gamma+2\left(v+\delta_{0}\right)=k+1}\left(A_{p}\right)_{\mu \gamma v \delta_{0}}(z, \xi)\left(2 i\langle z, \xi\rangle_{\ell}\right)^{v}=H(z, \xi) .
$$

Collecting terms in $(z, \xi)$ of bi-degree $(\alpha, \beta)$ with $\alpha+\beta=k+1-2 \delta_{0}$ in the above expression, we get

$$
\sum_{\theta=0}^{\alpha}\left(A_{p}\right)_{(\alpha-\theta)(\beta-\theta) \theta \delta_{0}}(z, \xi)\left(2 i\langle z, \xi\rangle_{\ell}\right)^{\theta}=H(z, \xi) .
$$

Recall $\left(A_{p}\right)_{\mu \gamma \nu \delta}=\sum_{j=1}^{N-n} \phi_{p, j}^{(\mu, v)}(z, 1) \bar{\phi}_{p . j}^{(\gamma, \delta)}(\xi, 1)$ by definition and $N-n<n-1$. Applying Lemma 5 to the above identity, one deduces $\left\|\left(A_{p}\right)_{\mu \gamma v \delta_{0}}\right\| \leq C$ for $\mu+\gamma+$ $2\left(v+\delta_{0}\right)=k+1$. This is a contradiction! Hence the claim holds.

The induction is thus complete. Consequently, for each $k,\left\|\phi_{p}^{(k)}\right\| \leq C$ with $C$ independent of $p$. We have shown for each fixed $k,\left\{\left\|F_{p_{j}}^{(k)}\right\|\right\}_{j=1}^{\infty}$ is bounded by some constant independent of $j$.

We are now in a position to prove Theorem 2 and Theorem 1 making use of the result in [MMZ].

Proof of Theorem 2, If $F$ is CR transversal to $M_{\ell}$ at 0 , then we are done. Assume $F$ is not CR transversal at 0 . Then there exists $p_{j} \rightarrow 0$ such that $F_{p_{j}}$ as constructed at the beginning of the section satisfies (12). Moreover, for each $k,\left\|F_{p_{j}}^{(k)}\right\| \leq C$ with $C$ independent of $j$ by Lemma 6. Following the same trick as in [HZ2], for each $k,\left\{F_{p_{j}}^{(k)}\right\}_{j=1}^{\infty}$ converges as $j \rightarrow \infty$ after passing to subsequences, to a certain $F^{*(k)}$. By the way these maps were constructed, the nontrivial formal map $F^{*}\left(=\left(f^{*}, \phi^{*}, g^{*}\right)\right):=\sum_{k} F^{*(k)}$ sends $M_{\ell}$ into $H_{\ell}^{N}$ satisfying the following normalization:

$$
\begin{gathered}
f^{*}(z, w)=z+o_{w t}(2), \\
\phi^{*}(z, w)=o_{w t}(1), \\
g^{*}(z, w)=w+o_{w t}(4) .
\end{gathered}
$$


According to a result of Meylan-Mir-Zaitsev [MMZ], the formal map $F^{*}$ is convergent. Hence, $F^{*}$ is a CR immersion at 0 sending $M_{\ell}$ into $H_{\ell}^{N}$.

Proof of Theorem 1; Assume by contradiction that $F$ neither is CR transversal to $M_{\ell}$ at 0 nor sends $U$ into $H_{\ell}^{N}$. Then there exists a CR immersion $F^{*}$ sending $M_{\ell}$ into $H_{\ell}^{N}$ by Theorem 2 On the other hand, since any two CR transversal maps between a Levi-nondegenerate hypersurface and a hyperquadric of the same signature differ only by an automorphism of the hyperquadric (see [EHZ]) when the codimension is less than $\frac{n-1}{2}$, there exists an automorphism $T$ of $H_{\ell}^{N}$ such that near $p_{j} \approx 0$, and hence at all points in $M_{\ell}$ near the origin,

$$
F=T \circ F^{*} .
$$

Since $T$ extends to an automorphism of the projective space $\mathbf{P}^{N}$ and $T(0)=0, F$ must be CR transversal at 0 . This is a contradiction.

Acknowledgements Part of work was done when the authors were attending the 7th workshop on geometric analysis of PDE and several complex variables at Serra Negra, Brazil. Both authors would like to thank the organizers for the kind invitation. The second author is also indebted to Wuhan University for the hospitality during her several visits there.

\section{References}

BER. M. S. Baouendi, P. Ebenfelt and L.P. Rothschild, Real submanifolds in complex space and their mappings, Princeton Mathematics Series, 47, Princeton University Press, Princeton, NJ, 1999.

BER2. M. S. Baouendi, P. Ebenfelt and L.P. Rothschild, Transversality of holomorphic mappings between real hypersurfaces in different dimensions. Comm. Anal. Geom. 15 (2007), no. 3, 589-611.

BH. M. S. Baouendi and X. Huang, Super-rigidity for holomorphic mappings between hyperquadrics with positive signature, J. Diff. Geom. 69(2005), 379-398.

BHR. M. S. Baouendi, X. Huang, and L.P. Rothschild, Nonvanishing of the differential of holomorphic mappings at boundary points. Math. Res. Lett. 2(1995), no. 6, 737-750.

BR. M. S. Baouendi and L.P. Rothschild, Geometric properties of mappings between hypersurfaces in complex space. J. Differential Geom. 31(1990), no. 2, 473-499.

CM. S. S. Chern and J. K. Moser, Real hypersurfaces in complex manifolds, Acta Math. 133(1974), 219-271.

Da. J. P. D'Angelo, Several complex variables and the geometry of real hypersurfaces. Studies in Advanced Mathematics, 1992.

EHZ. P. Ebenfelt, X. Huang and D. Zaitsev, The equivalence problem and rigidity for hypersurfaces embedded into hyperquadrics. Amer. J. Math. 127 (2005), no. 1, 169-191.

ER. P. Ebenfelt, L. R. Rothschild, Transversality of CR mappings. Amer. J. Math. 128, 1313-1343, (2006).

ES. P. Ebenfelt and D. Son, Transversality of holomorphic mappings between real hypersurfaces in complex spaces of different dimensions. Illinois J. Math. (56) (2012), no. 1, 33-51 (2013).

Fo. J. E. Fornaess, Biholomorphic mappings between weakly pseudoconvex domains. Pacific J. Math. 74(1978), 63-65. 
Iz1. A. Isaev, An estimate of the dimension of the image under a holomorphic mapping of real analytic hypersurfaces. Math USSR -Izv. 30 (1988), 89-102.

Iz2. A. Isaev, The image of Levi non-degenerate manifolds under holomorphic mappings, Complex Variables, 27 (1995), 217-233.

Hu. X. Huang, On a linearity problem for proper holomorphic maps between balls in complex spaces of different dimensions, J. Differential Geom. 51(1999), 13-33.

Hu2. X. Huang, Schwarz reflection principle in complex spaces of dimension two. Comm. Partial Differential Equations 21(1996), no. 11-12, 1781-1828.

HP. X. Huang and Y. Pan, Proper holomorphic mappings between real analytic domains in $\mathbf{C}^{n}$, Duke Math. J. 82 (1996), 437-446.

HZ. X. Huang and Y. Zhang, Monotonicity for the Chern-Moser-Weyl curvature tensor and CR embeddings. Sci. China Ser. A, 52(2009), no. 12, 2617-2627.

HZ2. X. Huang and Y. Zhang, On a CR transversality problem through the approach of the ChernMoser theory. J. Geom. Anal. 23(2013), no. 4, 1780-1793.

Mir. N. Mir, Convergence of formal embeddings between real-analytic hypersurfaces in codimension one, J. Differential Geom. 62 (2002), no. 1, 163-173.

MMZ. F. Meylan, N. Mir and D. Zaitsev, Approximation and convergence of formal CRmappings. Int. Math. Res. Not. 2003, no. 4, 211-242.

Pi. S. Pinchuk, Proper holomorphic maps of strictly pseudoconvex domains. (Russian) Sibirsk. Mat. Z. 15 (1974), 909-917, 959.

We. S. Webster, The rigidity of C-R hypersurfaces in a sphere. Indiana Univ. Math. J. 28 (1979), no. $3,405-416$. 